\title{
GÉNESIS DEL REAL DECRETO-LEY DE MARZO DE 1977
}

MIGUEL HERRERO DE MIÑÓN 


\title{
GÉNESIS DEL REAL DECRETO-LEY DE MARZO DE 1977
}

\author{
MIGUEL HERRERO DE MIÑÓN* \\ Letrado del Consejo de Estado
}

A la memoria de mi buen amigo Francisco Condomines, padrino de nuestra normativa electoral.

Conmemoramos los cuarenta años de las elecciones de 1977. Las primeras elecciones democráticas después de muchos años y las primeras limpias de toda nuestra historia constitucional. Unas elecciones organizadas de acuerdo con las normas electorales elaboradas, sobre las bases establecidas en la Ley para la Reforma Política de 1976 (Adicional Tercera), por un grupo de Secretarios Generales Técnicos del primer Gobierno Suárez y aprobadas en el mes de marzo de aquel año por el Real Decreto Ley 20/1977, de 18 de marzo, e incorporadas a la Constitución de 1978 (arts. 68 y 69).

Fue aquél un hito capital en el proceso de transición democrática, pero la suprema virtud de las normas de 1977, reiteradas en la Ley Electoral de 1985 consiste en que rompieron con una tradición de corrupción electoral que se remontaba a las Cortes de Cádiz y a las elecciones de 1813. Con ello se inaugura una práctica de limpieza electoral en que nadie, desde la izquierda o la derecha, desde el centro o la periferia, ha impugnado seriamente sus resultados fueran esos favorables o adversos.

¿Cuáles son las claves de tan exitosa ley? Primero, un sistema de representación proporcional asegurando de este modo que todas las grandes fuerzas políticas obtengan representación en todo el territorio y que lo hagan en proporción, sin duda mejorable, pero siempre suficiente, a los votos obtenidos. Segundo, fijar la provincia como circunscripción electoral, garantizando a todas ellas un mínimo de representación y evitando así la desertización política del territorio nacional. Tercero, un siste-

* Consejo de Estado. Calle Mayor, 79, 28013 Madrid. Email: consultas.consejo@consejoestado.es 
ma de filtros en la presentación de candidaturas y en la atribución de escaños. Ello favoreció la concentración del voto pasando del fraccionamiento tanto de la izquierda como de la derecha, la entonces llamada «sopa de letras», a grandes partidos homólogos a los del resto de la Europa democrática. Cuarto, la despolitización de la Administración electoral y la judicialización de su control. La normativa electoral posterior ha respetado estos cuatro principios fundamentales.

Creo que no es mal homenaje a norma tan criticada, antes y ahora, como exitosa en su práctica de cuatro décadas, el reconstruir la decantación de estos cuatro elementos nucleares.

En el otoño de 1976 nada de esto estaba claro. Ni para los reformistas del régimen a superar, ni para la llamada oposición democrática, ni para lo que entonces era la doctrina española en tales materias. Así lo prueba la escasa información sobre estos temas, no ya en la opinión pública, totalmente ajena a la cuestión, sino en los medios políticos y académicos. No faltaban viejos estudios españoles sobre los sistemas electorales. Dejando aparte el ensayo de Donoso Cortés ${ }^{1}$ sobre la Ley electoral de 1835 porque solo trata de la oposición entre elección directa o indirecta, durante la Restauración vieron la luz los Estudios sobre el Procedimiento Electoral de Navarro Amandi (Madrid, 1885) y el Tratado de Derecho Electoral Español de Sanmartín Puente (3 tomos, Madrid 1921), aparte de diferentes monografías sobre el derecho de sufragio como uno de los fundamentales derechos políticos. Más adelante Giménez Fernández introdujo una visión comparatista en sus Estudios de Derecho Electoral Contemporáneo (Sevilla, 1925) y Ortiz de Burgos se ocupó monográficamente de La Representación Proporcional (1921-1923, Madrid). En Cataluña la cuestión no deja de suscitar diversas opiniones, desde la de Rovira i Vigill (La representació proporcional en el sufragio universal, Barcelona 1910) a la serie de artículos en la Veu de Catalunya, de Ramón Marquina (1919, 9,10,12,14, 19 y 20 de agosto) y los de Trias de Bes (1936, 13, 15, 22 y 25 de marzo).

Sin embargo, frente a lo que ocurre en el resto de Europa, el interés por los sistemas electorales parece disminuir entre los académicos españoles a partir del comienzo del siglo xx. Es significativo que la Real Academia de Ciencias Morales y Políticas no le prestara atención y que mientras algunos líderes políticos hacen bandera del tema, la prestigiosa Revista de Derecho Público que vio la luz entre 1932 y 1936 no dedicó uno solo de los cincuenta y seis estudios monográficos en ella publicados a la cuestión electoral y solo hace excepción en tan pobre

${ }^{1}$ Donoso Cortés, J. (1970), Obras Completas, ed. BAC, tomo I, pp. 185 y ss. 
panorama el interés demostrado en favor de la representación proporcional por Pérez Serrano ${ }^{2}$.

Durante el régimen autoritario los sistemas electorales no fueron comprensiblemente el objeto preferido por los estudiosos españoles del derecho entonces llamado político y quienes intentaron, desde la década de los cincuenta, una apertura al comparatismo divulgaron las superficiales opiniones de Maurice Duverger ${ }^{3}$ sobre los efectos de los sistemas electorales en la configuración de los partidos políticos — simplificando, múltiples y rígidos, merced a la representación proporcional, bipartidismo, merced al mayoritario simple con distritos uninominales y multipartidismo flexible con el sistema mayoritario de doble vuelta- Es lógico que los reformistas del régimen, fueran tributarios de tales ideas. La traducción de alguna obra anglosajona ${ }^{4}$ no enriqueció mucho más el panorama.

Al hilo de la transición democrática, a pesar del bien estructurado y documentado estudio de Francesc de Carreras, Las Elecciones (Barcelona, Blume, 1977), la información no fue mucho mayor. Baste señalar que uno de los más acreditados académicos de re política, el Pfr. Sánchez Agesta ${ }^{5}$, en su monografía sobre la legislación electoral objeto del presente estudio, tan solo cita al propio Duverger y a D. Rae, cuya obra Leyes Electorales y sistema de partidos políticos (Madrid, CITEP,1977) acababa de ser traducida y publicada en español. Tampoco el llamado Documento Ollero, del que tanto se habló en aquellos años, dedicó una sola palabra a la cuestión electoral ${ }^{6}$.

El despertar de este «sueño dogmático» se encuentra en la fórmula electoral establecida diez años antes en la Conferencia Constitucional hispano guineana. De su segunda fase, en 1968, fui muy activo asesor, en colaboración con el diplomático Francisco Condomines, cualificado estudioso de los sistemas electorales, hasta mi exclusión cuando prevalecieron los criterios del Vicepresidente Carrero Blanco sobre los del Ministro de Exteriores, Castiella ${ }^{7}$.

2 Pérez Serrano, N. (1976), Tratado de Derecho Político, Madrid, Civitas, pp. 357 y ss. (el texto se escribe en los años 30); cfr. del mismo autor (1932), La Constitución española de 1931, Madrid.

3 Duverger, M. (1951), Les Partes Politiques, Paris (Colin) (trad. Esp. México, 1957) y RAE, D. (1977), Leyes electorales y sistema de partidos políticos, trad. esp, Madrid (CITEP). Cfr. Duverger, M. (1950), L'Influence des systèmes électoreaux sur la vie politique, Paris (Colin); Presses de Sciences Po, n. ${ }^{\circ} 16$.

4 Mackenzie, W. J. M. (1962), Free elections..., trad. esp., Tecnos.

5 SÁnchez Agesta, L. (1977), Ley Electoral. Introducción y Exposición, Madrid (EDERSA)

6 Texto en Herrero de MiÑón, M. (ed.) (1999), La transición democrática en España. Madrid (BBV), p. 99.

7 Cfr. Herrero de Miñón, M. (1993), Memorias de Estío, Barcelona, (Temas de Hoy), p. 28 y ss. Francisco Condomines que, junto conmigo, constituía el comité de asesoramiento técnico de 
Según las tesis de Duverger, mientras el sistema electoral mayoritario simple conduce al bipartidismo, el sistema proporcional fomenta la proliferación de partidos y evita las mayorías absolutas. Condomines mostró que el sistema mayoritario conduce al partido único si el sistema fuese de lista nacional y aun provincial y, en consecuencia, la alternativa podría ser otra que los distritos uninominales, muy convenientes cuando ya existe un sistema de partidos, pero que, en caso contrario, atomizarían la representación, eliminando cualquier gran fuerza política y dando el poder a los notables locales.

Todo eso parecía cierto y deseable para Guinea y Martín Villa, que como representante del Ministerio de Industria asistía a la conferencia, hizo patente ante su ministro, López Bravo, la incoherencia que suponía proponer para Guinea un sistema de partidos políticos que en España se consideraba vitando. Pero esta opción que, al final, fue fútil en Guinea Ecuatorial, resultó trascendental para España, y sus consecuencias llegan a la vigente Constitución y legislación electoral.

La cuestión se planteó definitivamente cuando el primer Gobierno de la Monarquía acomete la Transición. Pero los primeros proyectos elaborados al efecto sí insisten en el sufragio universal, directo y secreto para elegir las Cortes o, al menos la Cámara popular de las mismas, no parecen tener claro qué sistema electoral proponen. El proyecto Arias-Fraga guarda silencio al respecto ${ }^{8}$.

El «borrador sin padre» de ley de Reforma Política que el Presidente de las Cortes, Fernández Miranda entregó a Adolfo Suárez el 28 de Agosto de 1976, apenas aborda el tema en una escueta transitoria del siguiente tenor:

Se autoriza al Gobierno a regular por decreto-ley las primeras elecciones a Cortes; para las elecciones al Congreso de Diputados se ajustará al criterio de que cada partido legalmente inscrito obtenga un número de representantes proporcional al número de votos

la Conferencia, me convenció y convenció al Ministerio de Asuntos Exteriores que la organizaba, de las ventajas del sistema de representación proporcional con Ley d'Hont y listas completas cerradas y bloqueadas para obtener un sistema multipartidista sólido y facilitar el escrutinio y adjudicación de escaños. Así lo propuse al plenario de la Conferencia el 19 de abril de 1968 (Actas 1. ${ }^{a}$ sesión de la Segunda Fase de la Conferencia vid. Anejo Puntos básicos para el borrador de Constitución). A lo largo de las sesiones la fórmula fue apoyada por el partido nacionalista y modernizante (MONALIGE) (vid. Actas de la $14 .{ }^{a}$ sesión p. 28 y ss.) y combatida desde la Presidencia del Gobierno por el MUNGE y por el grupo pilotado por el Sr. García Trevijano y al final adoptada el 22 de junio de 1968 al término de la conferencia (vid. Actas de la 30. ${ }^{a}$ sesión de la Segunda fase de la Conferencia, p. 28-29). En mi archivo conservo un proyecto de ley electoral para el Estado de Guinea Ecuatorial expresamente aprobado por el entonces Ministro de Exteriores Castiella que es antecedente directo, y, en muchos artículos literal, de las normas de 1977. En Asuntos Exteriores el correspondiente archivo debe contener un ejemplar del mismo.

8 Texto en Herrero de Miñón, M. (ed.) (1999), La transición democrática..., op. cit., pp. 31 y ss. 
válidos obtenidos en las urnas. En las elecciones al Senado se estará a lo exigido por la naturaleza del mismo, que no permite la estricta proporcionalidad, pero sí criterio análogo de máxima objetividad.

El citado borrador de Ley para la Reforma Política fue muy reelaborado en el Ministerio de Justicia, pero el proyecto de Ley para la Reforma Política resultante cuyo cambio de preposición ha sido subrayado por su autor Landelino Lavilla $^{9}$, dio solo tímidos pasos en cuanto se refiere al sistema electoral. Los análisis políticos dirigidos por el propio Lavilla, el Vicepresidente Alfonso Osorio y, según parece Otero Novas, fueron muchos y en la Secretaría General del Ministerio de Justicia se avanzó en la redacción de un borrador de normas electorales sobre la base de circunscripción provincial y sistema proporcional. Pero en el texto del proyecto gubernamental de Ley para la Reforma enviado a las Cortes, la circunscripción electoral provincial quedó decidida respecto del Senado, no para el Congreso y la opción en pro de un sistema de representación proporcional tan solo apuntado de la siguiente manera:

Disposición transitoria primera: El Gobierno regulará las primeras elecciones a Cortes para constituir un Congreso de 350 diputados y elegir 204 senadores, a razón de cuatro por provincia, dos por Ceuta y dos por Melilla. Los senadores serán elegidos por sufragio universal directo y secreto de los españoles mayores de edad que residan en el respectivo territorio.

Las elecciones al Congreso se inspirarán en criterios de representación proporcional. Las elecciones al Senado se inspirarán en criterios de escrutinio mayoritario.

El informe del Consejo Nacional de 16 de Octubre de $1976^{10}$, repitiendo el criterio de Duverger, se pronunció en pro del sistema mayoritario, insistiendo en la pluralidad de versiones que cabían en el mismo.

Sin embargo, existe, prácticamente, un consenso doctrinal en considerar que el sistema mayoritario, que puede revestir un carácter puro y simple o atenuar sus efectos con el sistema a dos vueltas y estructurarse a través de escrutinio de lista o escrutinio uninominal tiende a la formación en las Cámaras de una mayoría homogénea que facilita la labor legislativa. El sistema proporcional tiene una aplicación técnica difícil de comprender por no poder determinarse a priori las consecuencias de los votos. Por ello, la representación proporcional puede fomentar un sistema de partidos múltiples e independientes, mientras que el escrutinio mayoritario a una sola vuelta tiende a la simplificación de partidos. El Consejo Nacional entiende que una ley Fundamental no debe pronunciarse sobre el sistema

9 Lavilla Alsina, L. (2017), Una historia para compartir, Madrid (Galaxia Gutemberg), p. 208.

10 Texto en Herrero de Miñón, M. (ed.) (1999), La transición democrática..., op. cit., pp. 119 y ss.

(C) UNED. Revista de Derecho Politico

N.o 100 , septiembre-diciembre 2017, págs. 33-48 
electoral aplicable, ni siquiera en sus disposiciones transitorias. Pero de cara a la futura Ley Electoral, cree recomendable el sistema mayoritario para ambas Cámaras, ya que solo a través de él puede lograrse un número no excesivo de opciones políticas que permitan el adecuado funcionamiento de la democracia.

A mi juicio fue en la tramitación en las Cortes del proyecto gubernamental donde se decidieron dos temas mayores; la opción de la provincia como circunscripción electoral y el sistema electoral proporcional.

El procedimiento de urgencia que en el Reglamento de las Cortes introdujo el Presidente Fernández Miranda en marzo de 1976, suprimía el trámite de comisión, de manera que podía pasarse directamente al Pleno desde la ponencia.

La documentación que ilustra este trámite obliga a desechar la interpretación de la gestación parlamentaria de la Ley para la Reforma Política como una mera escenificación de las previas decisiones gubernamentales. Tanto el Presidente del Gobierno como el de las Cortes veían en la activa intervención de éstas la piedra angular de la transición «de la ley a la ley»y, además, su campo privilegiado de acción. Ambos optaron por centrar allí las decisiones definitivas y Fernández Miranda mantuvo un eficaz control del procedimiento y monopolizó las relaciones entre el Presidente del Gobierno y la ponencia.

Fue en tal coyuntura cuando se planteó el grave tema del sistema electoral a seguir y cuyas bases pretendía fijar la Ley para la Reforma. Mientras que el proyecto gubernamental se había decantado por la proporcionalidad, los procuradores de la UPE, teledirigidos por Fraga desde fuera de la Cámara, insistían en el sistema mayoritario con circunscripciones uninominales cuyo mapa, cuestión harto difícil, estaba aún por hacer.

Los enmendantes citaban el ejemplo de Gran Bretaña y aducían las más que superficiales tesis de Duverger sobre la influencia de los sistemas electorales en la configuración del mapa político a las que ya hice referencia. Por parte del Gobierno y de la ponencia se argüía con la mayor exactitud de la representación proporcional, importante a la hora de conocer la realidad política española, y sobre la inconveniencia de las mayorías absolutas si es que éstas eran propiciadas artificialmente por el sistema electoral.

La ponencia, eficazmente dirigida por Fernando Suárez, examinó las más de trescientas enmiendas presentadas por los Procuradores, elaboró un largo y cuidadoso dictamen ${ }^{11}$ y todo ello pasó al Pleno en una maratoniana sesión los días 16 a 18 de noviembre de 1976.

11 Texto en Herrero de MiÑón, M. (ed.) (1999), La transición democrática ..., op. cit., p. 131 y ss. En p. 168 y ss. se incluye una síntesis del enjundioso debate en que los procuradores dieron 
La defensa del sistema mayoritario corrió a cargo del Sr. Martínez Esteruelas cuyo discurso puede resumirse en los siguientes párrafos.

Sólo me resta en este punto salir al paso de la tentación de que sean esgrimidos argumentos de tipo bistórico en favor de la representación proporcional, habida cuenta de su aplicación en abundantes países de Europa occidental. Únicamente he de decir a este respecto lo siguiente:

Primero. Supone un detalle curioso - yo afirmaría que extremadamente significativo_ que en las naciones de habla anglosajona — que son las que gozan de sistemas políticos tradicionalmente estables y democráticos-, la representación proporcional no se baya abierto camino.

Segundo. La representación proporcional solo se ha aplicado sin resultados insatisfactorios en pequeños países de Europa, como los reinos escandinavos, Bélgica y Holanda, en los que la estabilidad social derivada de su alto nivel de renta ha permitido compensar los defectos del multipartidismo. Pero pensemos que esta época de crisis económica en que toda vida política será más difícil, y es previsible que por mucho tiempo, puede poner de relieve que quienes se permitieron en la felicidad del auge económico el complicado sistema proporcional, tengan ocasión de arrepentirse de él.

Tercero. Es un hecho histórico — comúnmente aceptado como paradigma de las últimas ilógicas consecuencias a que puede llevarse la representación proporcional el fracaso de la República de Weimar, de 1919, sobre cuyo colapso y su desembocadura en.una férrea dictadura no es necesario que ilustre a los señores Procuradores.

Cuarto. Desde el término de la Segunda Guerra Mundial son muchos los países que, adoptando el sistema proporcional, pretenden el retorno al sistema mayoritario en alguna de sus modalidades. Francia ya lo bizo en 1958, tras la desdichada experiencia que proporcionó la IV República, y en la República Federal Alemana, donde impera un sistema de representación proporcional con amplias medidas correctoras, ha ganado terreno en la actualidad en la opinión pública la idea de abandonar dicho sistema y sustituirlo por otro mayoritario, a pesar de la oposición del partido liberal, que teme perder su cómoda posición de árbitro en la vida política alemana.

Quinto. La representación proporcional ha sido el sistema defendido en toda la Europa occidental por los respectivos partidos comunistas.

Sexto. $Y$, finalmente, si miramos a España, no es difícil pensar a qué nos conduciría la representación proporcional, aun con medidas correctoras - en cualquier caso, no se olvide que en ningún país se aplica el sistema proporcional puro e integral_-, habida cuenta de la proliferación increíble de partidos y siglas que ya estamos padeciendo. La

muestra de su ya reconocida competencia, hoy añorable, y de la libertad de criterio adquirida tras el cumplimiento de las previsiones sucesorias.

(C) UNED. Revista de Derecho Politico

N. ${ }^{\circ} 100$, septiembre-diciembre 2017, págs. 33-48 
experiencia bistórica nos ha enseñado suficientemente a qué ba conducido el multipartidismo en España ${ }^{12}$.

La opción proporcional, defendida por Fernando Suárez, venció y convenció. Sus palabras finales fueron:

En los escritos de observaciones generales de los señores Lostáu, Román, Morrondo García, así como en las enmiendas de los señores Muro Navarro, Gamazo Peláez, Rivas Guadilla, Fernández Nieto, Dancausa de Miguel, Zamanillo y González-Camino y señorita Plaza de Prado, se plantea un problema de indudable trascendencia, como es el de la distribución de los diputados del Congreso; se subrayan por los señores procuradores citados -con especial énfasis por parte del señor Morrondo García-las consecuencias a que podría llevar la distribución de los diputados con base en puros criterios de censo de población, señalándose cómo, caso de aceptarse estos criterios como exclusivos, una sola provincia podría contar con tantos diputados en el Congreso como cerca del $40 \%$ del territorio nacional; con el fin de evitar estos resultados, los señores procuradores citados proponen que se fije un cupo mínimo de diputados por provincia, distribuyéndose el resto en función del censo de población. La Ponencia manifiesta que los posibles desequilibrios a que aluden los señores procuradores están compensados por la existencia de un Senado de base exclusivamente territorial; por otra parte es de señalar que el proyecto no fija el ámbito de los distritos electorales ni la distribución de los escaños, por lo que nada impide que los problemas que preocupan a estos señores procuradores sean abordados en las normas electorales, que son las adecuadas para tratar esta materia, razón esta última por la que tampoco parece oportuno recoger la propuesta del señor Henríquez Hernández, relativa a la elección de un diputado por cada 100.000 babitantes.

Tal y como la Ponencia entiende que cumple su deber de mantener las líneas generales del proyecto y de aceptar opciones ofrecidas por el señor Martínez de Esteruelas, ese párrafo quedaría redactado así: "Las elecciones al Congreso se inspirarán en criterios de representación proporcional conforme a las siguientes bases: I) Se aplicarán dispositivos correctores para evitar fragmentaciones inconvenientes de la Cámara, a cuyo efecto se fijarán porcentajes mínimos de sufragios para acceder al Congreso; 2) La circunscripción electoral será la Provincia, fijándose un número mínimo inicial de diputados para cada una de ellas ${ }^{13}$.

Un tema especialmente polémico fue la atribución de senadores a las provincias insulares. En principio, prevaleció el criterio de mantener para cada una de ellas la misma cifra que para las provincias peninsulares, cuatro diputados y cuatro senadores a cada una. Sin embargo, el hecho insular y la diferencia de

\footnotetext{
${ }^{12}$ Ibid., pp. 248 y ss.

${ }^{13}$ Ibid., pp. 303.
} 
población entre unas y otras islas aconsejaba replantear la cuestión y así se hizo por los procuradores canarios en las Cortes, especialmente por el señor Olarte. En principio el Gobierno se negó a ello pero ante la insistencia que rayaba en lo dramático del procurador canario el principal ponente, Fernando Suárez, recurrió al Presidente de las Cortes y este organizó su entrevista con el Presidente del Gobierno al que convenció de la necesidad de introducir una diferencia en los archipiélagos ponderando la insularidad y la población. Para ello se mantuvieron los mismos diputados por cada provincia insular pero se añadió a cada una de ellas un senador más, lo que permitió a los redactores de las normas electorales a los que después me referiré, repartir dicho número atendiendo a la población de cada isla o grupo de islas menores.

Fraga, una vez más, cambió de criterio y desde el extranjero forzó a sus seguidores a apoyar la opción gubernamental de la ponencia. La transitoria primera de la ley quedó así:

El Gobierno regulará las primeras elecciones a Cortes para constituir un Congreso de trescientos cincuenta Diputados y elegir doscientos siete Senadores a razón de cuatro por provincia y uno más por cada provincia insular, dos por Ceuta y dos por Melilla. Los Senadores serán elegidos por sufragio universal, directo y secreto de los españoles mayores de edad que residan en el respectivo territorio.

Las elecciones al Congreso se inspirarán en criterios de representación proporcional, conforme a las siguientes bases:

Primera. Se aplicarán dispositivos correctores para evitar fragmentaciones inconvenientes de la Cámara, a cuyo efecto se fijarán porcentajes mínimos de sufragios para acceder al Congreso.

Segunda. La circunscripción electoral será la provincia, fijándose un número minimo inicial de Diputados para cada una de ellas.

Las elecciones al Senado se inspirarán en criterios de escrutinio mayoritario.

El Ministro Lavilla cerró el debate alegando dos razones políticas fundamentales en pro de la circunscripción provincial y la representación proporcional: Las entidades territoriales sobre las que se opera responden a la idea de la provincialización que es una realidad en nuestro régimen local y... nada mejor que en el recinto de la Cámara que ha de cobijar la representación nacional se encuentren presentes opciones no radicalmente opuestas y exclusivas sino repartidas a través del espectro politico en posiciones capaces de llegar fácilmente o más fácilmente al pacto y la colaboración ${ }^{14}$.

A partir del 20 de diciembre el Gobierno designó un comité constituido por los Secretarios Generales de Justicia (Herrero de Miñón), Interior (Santamaría)

14 Ibid., pp. 309-310. 
y Presidencia (Vizcaíno), a los que se añadieron algunos funcionarios de este departamento y, a propuesta mía, el Vicesecretario General Técnico de Exteriores, Francisco Condomines, ya mencionado. Para compensar los celos ministeriales, se acordó que las reuniones fueran en Castellana 3 y que Herrero llevara la ponencia y el criterio político fijado por el gobierno. Los datos estadísticos los proporcionaba Juan Santamaría, los funcionarios de Presidencia, dirigidos por Miguel Vizcaíno, la logística, y Condomines asumía la asesoría técnica.

El primer problema que se planteó fue el de la forma de promulgación de la nueva normativa electoral. Bien como un supuesto de legislación delegada por entender que la citada transitoria primera de la Ley para la Reforma Política constituía una delegación como las contempladas en el artículo 51 de las entonces vigentes Ley Orgánica del Estado y 10.4 de la Ley de Régimen Jurídico de la Administración del Estado, bien un Decreto-Ley de acuerdo con las previsiones del artículo 13 de la Ley de Cortes. La primera fórmula requería un dictamen preceptivo de la Comisión Permanente del Consejo de Estado y podía temerse que en aquel momento, dada su composición, no fuera muy favorable a la normativa reformista. Y otro tanto ocurría con la correspondiente comisión de las Cortes prevista en el citado artículo 13. El Presidente Fernández Miranda aseguró que garantizaba la posición favorable de la correspondiente comisión de las Cortes y así se hizo con el resultado positivo de todos conocido.

En cuanto a las cuestiones de fondo, Alfonso Osorio, sobre la base de la documentación que la Comisión de Secretarios Generales Técnicos envió al Gobierno y de su personal experiencia en la gestión del asunto, hizo una escrupulosa síntesis del trabajo de los redactores y de los avatares de este trámite ${ }^{15}$.

Sirvió de base inicial del grupo de trabajo el proyecto elaborado en el Ministerio del Interior del primer gobierno de la Monarquía que aportó el señor Santamaría. La introducción de los nuevos criterios electorales para dos diferentes cámaras fijados por la Ley para la Reforma Política y la ya creciente liberalización política española, exigió profundas modificaciones en el citado borrador que sin embargo fue aprovechado como documento de base. Dicho proyecto era a su vez, tributario de la entonces vigente ley electoral de 1907 que, en consecuencia, es el texto que cuantitativamente ha influido más en las normas electorales de 1977. También se tuvo en cuenta el Decreto 1796/1967, de 20 de julio, por el que se dictan normas complementarias de la Ley de Representación Familiar en las Cortes. En cuanto al derecho comparado, estuvo siempre presente en los trabajos de redacción. La normativa electoral francesa influyó en lo relativo a incompati-

15 Osorio, A. (1980), Trayectoria política de un Ministro de la Corona, Barcelona (Planeta), p. 169 y ss. 
bilidades e ineligibilidades; la portuguesa de 1974 en los artículos 45 a 48, e incluso la japonesa. La práctica anglosajona de neutralización política de la administración electoral llevó a la objetivación y judicialización de la misma y al cambio de denominación de juntas del censo por juntas electorales. Dos libros fueron fundamentales en esta ocasión como lo habían sido en el antecedente guineano. Ambos de Smith: Free elections in the Developping Countries (Londres 1960) y The New Commanwelth and its constitutions (Oxford 1964).

Problema grave en el desarrollo de la Disposición Transitoria Primera de la Ley para la Reforma Política, fue la distribución de los diputados entre las demarcaciones provinciales respetando un mínimo común a todas ellas y sin perjuicio de responder al criterio de «representación por población», esto es, número de diputados en función de la densidad demográfica. Se manejaron por los encargados de la elaboración de la norma varias alternativas y se llegó a la conclusión de que la fórmula más adecuada, atendiendo a los datos del censo, era la fijación de un diputado por cada 108.000 habitantes, asegurando en todo caso a las provincias un mínimo de cuatro. Este criterio permitía evitar cualquier injusticia en la distribución de diputados por población, al no privar a ninguna provincia de ningún diputado y permitir, mediante la gratuita distribución de los 43 diputados sobrantes, que todas las provincias tengan como mínimo 4 diputados, cifra a partir de la cual funciona con bastante regularidad el escrutinio de lista y la representación proporcional. Es evidente que, con este sistema, Barcelona alcanza el número excesivo de 41 diputados y Madrid el de 40, cifras tan grandes que no resultan convenientes a ningún efecto.

Por eso, en la redacción final se rebajó a tres el número mínimo de diputados por provincia por considerar que el de cuatro igualaba injustamente a las provincias pequeñas con las medias.

Al estudiar los porcentajes mínimos de votos para tener acceso a la Cámara se barajaron varios criterios, fundamentalmente reducibles a dos sistemas: las diversas fórmulas de distribución de restos y el mínimo estéril.

El mínimo estéril a nivel nacional perjudicaba gravemente a las fuerzas políticas regionales y a nivel provincial solo sería admisible si no supusiera en las circunscripciones más populosas, la esterilización de un número de votos comparativamente muy superior al exigido para obtener votos en una circunscripción menos poblada, v. gr. un coeficiente del $5 \%$ (sistema alemán) aplicado en Madrid supondría la esterilización de 100.000 votos o más, cifra equivalente a la totalidad del electorado en varias provincias.

Por ello, se estimó más conveniente como correctivo eficaz y equitativo a la distribución de restos, el sistema del mayor resto. Este sistema, permite fortalecer a los grandes partidos a la vez que no incide en la esterilización mecánica de 
un porcentaje fijo de votos. Sin embargo, para respetar la letra de la Ley para la Reforma Política, se incluyó un mínimo estéril muy reducido, concretamente el $3 \%$, que nunca excediese al cociente electoral, tomando como caso ejemplar a las grandes poblaciones.

De acuerdo con el criterio de Condomines que, tras largas explicaciones, el Gobierno ya había hecho suyo, el sistema era el de la ley d'Hont que no necesaria pero sí lógicamente llevaba a una fórmula de listas completas cerradas y bloqueadas. El Vicepresidente Osorio manifestó su desacuerdo con dicha fórmula que, a su juicio, entregaba la suerte de los candidatos a los estados mayores de los partidos políticos y no a la preferencia de los electores. Lo cierto es que entonces, arguyó Lavilla, se trataba de consolidar los partidos y conseguir un sistema de escrutinio sencillo. Ambos tuvieron razón. Las normas de 1977 normalizaron el mapa político reduciendo el número y consolidando la estructura de los partidos. Pero, al convertir en definitivo lo ideado como provisional, han contribuido paulatinamente a hacer de los parlamentarios émulos de «Juan empleado, de profesión temblador», caricatura decimonónica del funcionario todavía no profesional angustiado por la cesantía. Por otra parte, al decir del entonces Vicepresidente, Adolfo Suárez quería controlar las listas y el resultado $^{16}$. Si la ley electoral se reformase éste sería el primer extremo a corregir.

En cuanto a la elección del Senado, el problema consiste en la articulación de los criterios mayoritarios a que se refiere la Ley para la Reforma Política. Entre las múltiples fórmulas que pueden utilizarse al respecto — sufragio mayoritario de lista, voto único transferible, voto único no transferible, sufragio restringido, etc.- - pareció conveniente adoptar el voto único restringido, que, sin dejar de responder a los principios del sistema mayoritario, incorpora indirectamente un elemento de proporcionalidad. Sus rasgos fundamentales son: en circunscripciones pluripersonales, se confiere a cada elector un único voto sobre un número de candidatos menor del propuesto. Sin embargo, en una sola vuelta, han de resultar cubiertos todos los escaños que correspondan a la circunscripción.

Cuestión aparentemente no tan polémicas pero de extraordinaria importancia fue la plena judicialización de la administración electoral que tiende a relajarse pero se mantiene después en la ley de 1985. Así se sustituyó en las juntas provinciales al secretario de la Diputación correspondiente por el secretario de la Audiencia y se introdujo una muy amplia mayoría de personal judicial en las juntas de diverso nivel.

16 Ibid., p. 271, y Lavilla Alsina, L., op. cit., p. 325 y ss. Una prueba de esta pretensión del Presidente Suárez en Herrero de Miñón, M., Memorias de Estío, op. cit., p. 162. 
El contencioso electoral ofrecía tres posibilidades, la solución parlamentaria que atribuye a cada Cámara el enjuiciamiento de esta materia y que la práctica española de 1931 a 1936 demostró que se presta a una excesiva politización, la solución judicial, que ya había pretendido Bravo Murillo en España como remedio al sectarismo partidista, y el sistema mixto previsto en la ley de 1907, que remitía la resolución a las Cámaras previo dictamen del Tribunal Supremo. Los redactores del texto de 1977 optaron por la total judicialización del sistema y articularon el mismo atendiendo a la ley de jurisdicción contenciosa entonces vigente debidamente simplificada.

En cuanto a la campaña electoral, se acortó a iniciativa de Lavilla, a 21 días.

Redactado el proyecto, los Secretarios Generales Técnicos redactores se reunieron bajo la presidencia del entonces Subsecretario del Presidente del Gobierno, Sr. Otero Novas, durante dos jornadas con tres delegados de la entonces oposición, especialmente los Sres. Galeote, por el PSOE; Bobillo, por el PSP, y Arias Salgado, por la entonces llamada Socialdemocracia, para discutir y, en su caso, pactar la norma. Los citados opositores coincidían tanto en la circunscripción provincial como en la representación proporcional en el Congreso y el mayoritario con voto restringido en el Senado, pero insistían en desjudicializar un tanto el contencioso electoral y en permitir la presentación de los candidatos por más de una circunscripción a efectos de que los líderes de los partidos especialmente populares pudieran arrastrar el voto en diferentes circunscripciones. Ninguna de estas pretensiones fue aceptada.

Title:

Genesis of the Royal Decree of March 1977.

\section{Resumen:}

En este trabajo se exponen, con elevado nivel de detalle, los elementos que influyeron en la redacción del Real Decreto Ley de 18 de marzo de 1977, que reguló las primeras elecciones democráticas en España, y que influyó posteriormente en la actual regulación electoral todavía vigente. 


\begin{abstract}
In this work are exposed, with high level of detail, the elements that influenced the drafting of the Royal Decree of March 18, 1977, which regulated the first democratic elections in Spain, and which later influenced the current electoral regulation still in force.
\end{abstract}

\title{
Palabras clave
}

Transición española; Elecciones; sistema electoral.

Key words

Spanish transition; Elections; electoral system. 